\title{
Genes WHEAT FRIZZY PANICLE and SHAM RAMIFICATION 2 independently regulate differentiation of floral meristems in wheat
}

Oxana B. Dobrovolskaya ${ }^{1,2^{*}}$, Yumiko Amagai ${ }^{3}$, Karina I. Popova ${ }^{1}$, Alina E. Dresvyannikova ${ }^{1,2}$, Petr Martinek ${ }^{4}$, Alexander A. Krasnikov ${ }^{5}$ and Nobuyoshi Watanabe ${ }^{3}$

From Belyaev Conference

Novosibirsk, Russia. 07-10 August 2017

\begin{abstract}
Background: Inflorescences of wheat species, spikes, are characteristically unbranched and bear one sessile spikelet at a spike rachis node. Development of supernumerary spikelets (SSs) at rachis nodes or on the extended rachillas is abnormal. Various wheat morphotypes with altered spike morphology, associated with the development of SSs, present an important genetic resource for studies on genetic regulation of wheat inflorescence development.

Results: Here we characterized diploid and tetraploid wheat lines of various non-standard spike morphotypes, which allowed for identification of a new mutant allele of the WHEAT FRIZZY PANICLE (WFZP) gene that determines spike branching in diploid wheat Ttiticum monococcum L. Moreover, we found that the development of SSs and spike branching in wheat $T$. durum Desf. was a result of a wfzp-AVTtBH-A1 mutation that originated from spontaneous hybridization with T. turgidum convar. compositum (L.f.) Filat. Detailed characterization of the false-true ramification phenotype controlled by the recessive sham ramification 2 (shr2) gene in tetraploid wheat T. turgidum L. allowed us to suggest putative functions of the SHR2 gene that may be involved in the regulation of spikelet meristem fate and in specification of floret meristems. The results of a gene interaction test suggested that genes WFZP and SHR2 function independently in different processes during spikelet development, whereas another spike ramification gene(s) interact(s) with SHR2 and share(s) common functions.

Conclusions: SS mutants represent an important genetic tool for research on the development of the wheat spikelet and for identification of genes that control meristem activities. Further studies on different non-standard SS morphotypes and wheat lines with altered spike morphology will allow researchers to identify new genes that control meristem identity and determinacy, to elucidate the interaction between the genes, and to understand how these genes, acting in concert, regulate the development of the wheat spike.
\end{abstract}

Keywords: Frizzy panicle, Sham ramification 2, Spike branching, False-true ramification, Grasses, Wheat, Spike, Meristem, Inflorescence development

\footnotetext{
* Correspondence: oxanad@bionet.nsc.ru

${ }^{1}$ Institute of Cytology and Genetics, SB RAS, Lavrenvieva ave. 10, Novosibirsk 630090, Russia

${ }^{2}$ Novosibirsk State University, Pirogova, 2, Novosibirsk 630090, Russia

Full list of author information is available at the end of the article
} 


\section{Background}

The flower is the most important reproductive organ of angiosperms, and is directly related to the processes of pollination, fertilization, development of the embryo, and the formation of seeds and fruits. Cereal flowers, florets, develop on a specialized short branch called a spikelet. This is the basal unit of the cereal inflorescence and is a characteristic of all modern grasses, except for only one early-diverged lineage [1]. Spikelets may develop either directly on the inflorescence axis or from primary or secondary inflorescence axillary meristems. The pattern of branching and specification of spikelet meristems determines the architecture of the cereal inflorescence. Wheat inflorescence, the spike, is characteristically unbranched, and sessile spikelets are directly attached to the spike axis (rachis) at rachis nodes. Spikelets consist of two glumes and 2-5 fertile florets arranged along an indeterminate axis, the spikelet rachilla. The number of spikelets per a rachis node is a key taxonomic characteristic of the Triticeae tribe $[2,3]$. In all wheats, including diploid Triticum monococcum L. $(2 n=2 \times=14$, genome formula AA), tetraploid T. durum Desf. ( $2 n=2 \times=28$, BBAA), and hexaploid T. aestivum $\mathrm{L}$. $(2 n=2 \times=42$, BBAADD) species, normally one spikelet develops at a spikelet node and formation of supernumerary spikelets (SSes) or branch-like structures is considered an abnormality and observed rarely. The exception is tetraploid wheat Triticum turgidum L. Forms of T. turgidum with spike branching have been known for 2000 years; Pliny the Elder mentioned them under the name ramosum and centigranum (23-79 AD) [4]. Because of the tendency to form branching spikes, this tetraploid wheat has been variously called e.g. "Miracle wheat", "Mummy", "Egyptian", "Jerusalem wheat", "Seven-headed" $[4,5]$. Now T. turgidum forms with branching spikes are classified as T. turgidum convar. compositum (L.f) A. Filat. All T. turgidum branching forms have a common spike phenotype: additional spikelets develop on branches and directly at rachis nodes in the lower one-third of the spike; this spike phenotype is often referred to as the turgidum type of branching. In another tetraploid species, T. durum Desf. (BBAA), forms with spike branching are rare $[4,6]$. Coffman [7] has described spontaneous mutants with SSes that have been found in the Mindum durum wheat variety. These mutants are characterized by the occurrence of one or two additional sessile spikelets at several rachis nodes in the lower part of the spike; the phenotype is different from the turgidum spike branching. Spontaneous mutant forms with spike branching have been found by M.M. Jakubziner in two T. durum cultivars: Akmolinka 5 and Hordeiforme [8]. V.F. Dorofeev has found $T$. durum forms with a spike branching phenotype similar to the turgidum type of branching in local mixed populations of tetraploid ( $T$. durum, $T$. turgidum) and hexaploid (T. aestivum) wheat species grown in Transcaucasia [8]. Forms with spike branching and sessile SSes have also been found in tetraploid T. dicoccum (Schrank) Schuebl. and occasionally in T. polonicum L. species [6].

In diploid wheat species, spontaneous mutants with spike branching have not been reported [9], whereas an induced mutant that is characterized by the development of SSes at rachis nodes and on extended spikelet rachilla (on branches) was obtained by Dr. Yamashita in the second half of the last century [10].

In hexaploid bread wheat T. aestivum L., SS forms, including spike branching forms, are rare. Percival [6] described forms with sessile SSes in bread wheat, whose spike phenotypes are similar to that of the spontaneous mutant of the Mindum durum wheat variety [7]. Forms with the SS phenotype have been obtained as a result of intra- and interspecific hybridization, treatment with radioactive isotopes, or chemical mutagens, and spontaneous mutagenesis [11-14]. Martinek and Bednař [15, 16] have recorded several bread wheat morphotypes with SSes: multirow spike (MRS, a large number of spikelets emerge from each rachis node in the lower one-third of the spike); horizontal spikelets (HS), syn. 'tetrastichon sessile spikelets'(two or three spikelets are in a horizontal position at a spike rachis node); vertical or pared spikelets, (VS, two spikelets arise vertically at a spike rachis node), this phenotype also called banana or tween spikelets; and genuine branching (GB), or ramified spike (RS), in addition to the sessile SSes, lateral branches bearing spikelets are formed in the basal part of the spike. This group of SS lines also includes the triple spikelet phenotype of Tibetan triple-spikelet wheat [14], as a type of HS. The GB spike phenotype of hexaploid wheat is similar to the turgidum type branching, thus, the terms genuine branching, turgidum type of branching and ramified spike or true spike ramification are used as synonyms. Four-rowed spike that was first recorded in tetraploid T. turgidum wheat by Klindworth et al. [17] is a type of HS. In addition to the "true spike ramification", the term "false-true spike ramification" was recently proposed to describe a particular type of an SS phenotype, that is characterized by elongation of the spikelet rachilla, which bears two florets at basal nodes and SSes at distal nodes [18].

In 1910, E. von Tschermak reported that the spike branching phenotype of T. turgidum is under monogenic recessive control, and the gene was designated as $b h$, branched head [6]. Results of further research confirmed the recessive mode of inheritance of SS/spike branching in tetraploid T. turgidum and T. durum wheat species, and revealed the same type of inheritance in diploid $T$. monococcum and hexaploid T. aestivum [10, 19-22]. The expression of the trait is modulated by a number of environmental factors $[11,19]$. The loci that determined 
the SS/spike branching phenotype have been localized on wheat chromosomes of homoeologous group two: $2 \mathrm{~A}^{\mathrm{m}}$ in diploid [10], 2A in tetraploid [21, 23], and 2A and $2 \mathrm{D}$ in hexaploid wheat $[20,24-26]$. The formation of SSes in hexaploid bread wheat is controlled by homoeologous genes WHEAT FRIZZY PANICLE (WFZP) located on chromosome arms 2AS, 2BS and 2DS, respectively [25]. The structural and functional characterization of the three WFZP homoeologous genes has revealed that mutations of WFZP-D and WFZP-A caused development of supernumerary spikelet phenotypes of MRS, HS, and RS morphotypes in hexaploid bread wheat lines. Poursarebani et al. [27] has suggested that a single amino acid substitution in the AP2/ERF domain of TtBH-A1, which represents a mutant allele at the WFZP-A locus, causes spike branching in tetraploid $T$. turgidum wheat. In addition to WFZP-A/TtBH-A1, other genetic factors are involved in the control of spike branching of the turgidum type [28, 29]. Although the TtBH-A1/wfzp-A mutant allele at the WFZP-A locus is the main genetic determinant of the turgidum type of branching/true spike ramification in tetraploid wheat, "false-true spike ramification" phenotype of $T$. turgidum is under the control of a recessive allele at the single SHR2 locus (SHAM RAMIFICATION 2) [30]. The shr2 gene was placed to chromosome 2AL by genetic mapping [30], but its structure is not defined so far. The "true spike ramification" and "false-true spike ramification" phenotypes have some similarities, development of SSes on an extended rachilla, but it is not clear whether genes $w f z p$ and shr2 are involved in the control of the same developmental processes.

Here, we characterized mutants of diploid wheat $T$. monococcum and tetraploid wheat $T$. durum that show SS/true spike ramification phenotypes; we also identified causative mutations at the WFZP-A gene locus, including a novel $w f z p-A .2$ mutation, and demonstrated that that the wfzp-A/TtBH-A1 allele may be transferred to $T$. durum via spontaneous hybridization with $T$. turgidum. Moreover, using SEM analysis, we revealed the characteristic features of inflorescence development of the "false-true spike ramification" morphotype and suggested putative functions of the SHR2 gene. A possible interaction between genes WFZP and SHR2, which determine the two spike branching phenotypes, was investigated based on evaluation of $F_{2}$ hybrid phenotypes.

\section{Methods}

\section{Plant material}

Diploid wheat material. KT $3-24$ is a branched spike induced mutant of T. monococcum L. $(2 n=2 \times=14$, genome formula $\mathrm{A}^{\mathrm{m}} \mathrm{A}^{\mathrm{m}}$ ) developed by Dr. K. Yamashita [10]. T. sinskajae A. Filat. et Kurk. $(2 n=2 \times=14$, genome formula
$\mathrm{A}^{\mathrm{m}} \mathrm{A}^{\mathrm{m}}$ ) accession PI 418587 is characterized by nonbranched soft and semi-compact spike. KT 3-24 and PI 418587 were the parents of the $F_{2}$ mapping population for molecular-genetic mapping of the $b h^{m}$ gene that determined the mutant spike phenotype [10].

Tetraploid wheat material. T. durum Desf. var. ramosoobscurum Jakubz. $(2 n=2 \times=28$, genome formula BBAA) "Vetvistokolosaya" R-107 (R-107 throughout the paper), a semi-dwarf and branched spike accession, is a natural mutant collected in Dagestan, Russian Federation. Branched spike phenotype of R-107 is under the control of a recessive allele at a single gene locus $b h$, located on chromosome 2AS [21].

T. turgidum L. K-40750 $(2 n=2 \times=28, \mathrm{BBAA})$ is a branched accession of $T$. turgidum var. plinianum originated from Bulgaria and provided by the N.I. Vavilov All-Russian Institute of Plant Genetic Resources (St. Petersburg, Russia).

T. turgidum L. PI $67339(2 \mathrm{n}=2 \times=28$, BBAA $)$ is a tetraploid wheat accession with false-true ramified spike provided by the National Small Grain Collection (NSGC).

T. durum Desf. $(2 n=2 \times=28$, BBAA) Langdon 222 (LD222 throughout the paper) is a standard spiked accession.

We produced three $F_{2}$ populations: (1) R107/K-40750 to assess the allelic relationship of genes that determine turgidum type of spike branching (K-40750) and SS/RS phenotype (R-107); this population consisted of 104 plants; (2) PI 67339/R-107 and (3) PI 67339/K-40750 to test possible interaction between the genes that determine branching phenotypes of different types. Populations \#2 and \#3 consisted of 143 and 162 individuals respectively. $F_{1}$ and $F_{2}$ hybrids were grown under greenhouse conditions of IC\&G SB RAS, Novosibirsk, Russia. Spike and spikelet morphology was observed after heading.

\section{SEM analysis}

Developing spikes from lines/accessions with the SS phenotype (KT 3-24, R-107, K-40750, PI 67339) and normal spiked lines, LD222 and PI 418587, were dissected with a scalpel under a binocular microscope (Altami PS0745, "Altami", St. Petersburg, Russia). Scanning electron microscope, SEM (TM-1000, Hitachi Co. Hitachi, Ltd., Japan), was used to observe the morphological features of the inflorescences as described in Dobrovolskaya et al. [25]. Dissected young spikes were examined under low vacuum conditions (30-50 $\mathrm{Pa}$ ) and an accelerating voltage of $15 \mathrm{kV}$.

\section{Sequencing}

Sequences of the WFZP-A gene (up to $3000 \mathrm{bp}$, including the coding part of the genes, promoter regions and 3'-regions) of KT 3-24, R-107, K-407750 (SS/true spike ramification), normal spiked LD222 and PI 418587 lines 
were obtained by direct Sanger sequencing of PCR products using the BigDye Terminator v3.1 cycle sequencing kit (Applied Biosystems), following the manufacturer's instructions. Fluorescently terminated extension products were separated using a capillary ABI-3730 Bioanalyzer (Applied Biosystems). All sequences obtained in this study are included in Additional file 1.

All primers used in this study are listed in Table 1.

\section{Mapping procedure}

A previously obtained $\mathrm{F}_{2}$ mapping population [10] were genotyped using a $w f z p$-SSR marker, ssrCS248B13-1 [31]. The SSR analysis was performed as described in Dobrovolskaya et al. [25]. The $w f z p-A .2$ gene was integrated into $2 \mathrm{~A}^{\mathrm{m}}$ genetic map reported by Amagai et al. [10].

\section{Results}

Standard wheat inflorescence, the spike, consists of sessile spikelets that are directly attached to the spike rachis in the distichous order, one spikelet per a rachis node. The spikelet is a short branch, bearing florets; a spikelet consists of two glumes and 2-5 florets arranged along a spikelet rachilla. The main axis in wheat is determinant and terminates with a terminal spikelet, although in diploid T. monococcum, the terminal spikelet is rudimentary or missing [32-34]. Here, we characterized lines and accessions of diploid and tetraploid wheats with nonstandard spike morphology that are characterized by the development of SSes and/or extension and branching of a spikelet rachilla. The SS group is heterogeneous and comprises lines with different morphotypes: horizontal spikelets, genuine branching, and false-true spike ramification (Figs. 1, 2, 3).

Table 1 Primers used for the WFZP-A sequencing and genotyping

\begin{tabular}{|c|c|c|}
\hline Primer Name & Sequence $5^{\prime}-3^{\prime}$ & Location \\
\hline \multicolumn{3}{|c|}{ Amplicon sequencing } \\
\hline WFZP_2A_F1 ${ }^{a}$ & CATGGGCAAATCGGTTAATG & 5 'region \\
\hline WFZP_2A_R $1^{a}$ & TGGATGAGATGGCGAGGTAG & \\
\hline WFZP_F2 ${ }^{a}$ & TCTTGTCAGTGGCAGGCATC & $5^{\prime}$ region \\
\hline WFZP_2A_R2 ${ }^{a}$ & TGGCAGAAGTGAAGTGAGGT & \\
\hline WFZP_F3 ${ }^{a}$ & GCTCACAGTCTCAGCAACCA & 5'-UTR -CDS \\
\hline WFZP_2A_R3 ${ }^{a}$ & CACTGGGCACCGGCATGGAA & \\
\hline WFZP_2AD_F4 ${ }^{\mathrm{a}}$ & CAGCCAACCTCACTTCACT & CDS \\
\hline WFZP_2A_R4 ${ }^{a}$ & GCTAGGGCACCGAAACAAC & \\
\hline WFZP_F5 ${ }^{a}$ & ACGACATGGTCGCCTCGT & CDS-3' region \\
\hline WFZP_2A_R $5^{a}$ & GGATCGGGGTGGATAGATTG & \\
\hline \multicolumn{3}{|l|}{ SSR genotyping } \\
\hline $\operatorname{ssrCS} 248 \mathrm{~B} 13-1 \mathrm{~F}^{\mathrm{b}}$ & CTCCAAGAAGATCGAGGTGAACAT & \\
\hline $\operatorname{ssrCS} 248 \mathrm{~B} 13-1 \mathrm{R}^{\mathrm{b}}$ & TTGTTACCCTACCGATGATGTGTG & \\
\hline
\end{tabular}

\section{HS (horizontal spikelets) and true spike ramification/} genuine branching phenotypes

Diploid T. monococcum L. wheat. KT 3-24, an induced fertile mutant of T. monococcum, is characterized by a genetically stable SS trait. Additional spikelets developed on short branches (Fig. 1a). At the earliest stage of development, KT 3-24 inflorescence developed normally (Fig. 1e); inflorescence meristem produced primary axillary meristems (AxMs) that first initiated glume primordia and then gave rise to secondary AxMs (Fig. 1g). In the wild type (WT), secondary AxM (floral meristem in a WT inflorescence) first produced lemma and palea, and then formed floral organs (Fig. 1d, f), but in KT 324 inflorescence, secondary AxMs formed glume primordia (Fig. 1f) and then generated the other spikelet organs (Fig. 1j), and thus ectopic spikelets developed at the location of florets of a primary spikelet. Several secondary AxMs of a primary spikelet developed into ectopic spikelets and, as a result, a branch-like structure formed. Glumes of primary spikelets remained rudimentary and could be distinguished only at early stages of spike development (Fig. 1h, j). Ectopic spikelets developed as WT spikelets and produced florets. The meristems of ectopic spikelets formed at $90^{\circ}$ to normal spikelets.

The feature of the KT-3-24 inflorescence development were similar to those of previously described bread wheat SS mutants [25] and bh1 mutants of $T$. turgidum [27], namely, 1) development of an ectopic spikelet at the location of the floret of the primary spikelet; 2) development of ectopic spikelet meristems at $90^{\circ}$ to normal spikelets; 3) in an ectopic branch-like structure, the basal florets of the primary spikelet were always replaced by ectopic spikelets; 4) rudimentary glumes of the primary spikelets were visible only at the early stages of inflorescence development.

Suggesting these features of inflorescence development together with the location of gene $b h^{m}$ responsible for the mutant phenotype on the 2AS molecular-genetic map [10], we hypothesized that $b h^{m}$ is a mutant allele at the WFZP gene locus in diploid T. monococcum wheat. A set of WFZP-A sequence-specific primers [25] were used for sequencing of WFZP in T. monococcum (Table 1). In line KT 3-24, a non-synonymous substitution, TGC (Cys) to TAC (Thr), was found in the AP2/ERF functional domain of WFZP (Fig. 1b). The WFZP sequences of $F_{2}$ individuals from the KT 3-24/PI 418587 cross were determined. It was found that all the plants with the mutant phenotype had the mutant allele. Moreover, the CS248B13 microsatellite marker (Table 1) developed on the basis of sequence analysis of the WFZP-A locus [31] was applied to genotype the $\mathrm{F}_{2}$ plants. WFZP and CS248B13 were mapped on chromosome 2AS map and they did not recombine with $b h^{m}$ based on $94 \mathrm{~F}_{2}$ individuals (Fig. 1c). Thus, the mutant 


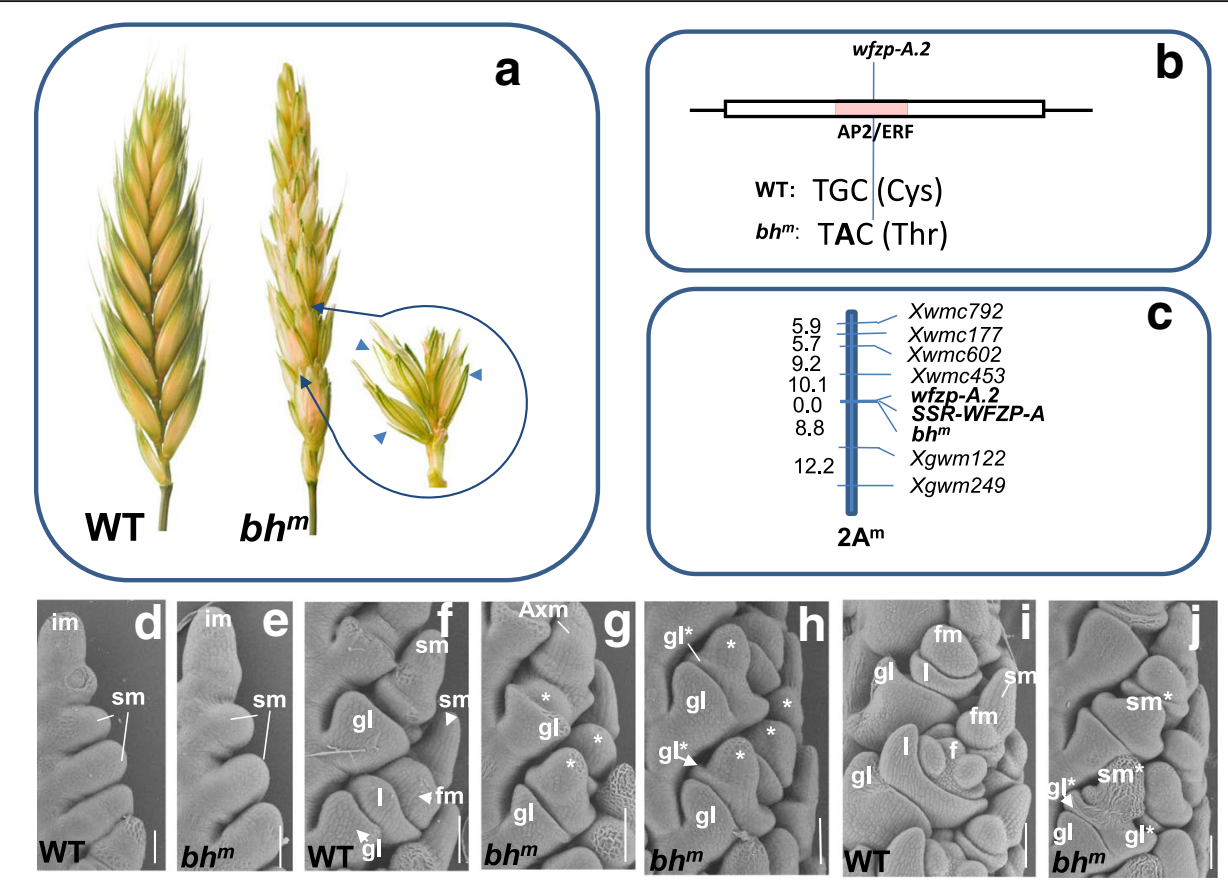

Fig. 1 Spike branching in diploid T. monococcum wheat. a, Illustration of the spike structure in the T. monococcum KT 3-24 (middle), harboring short branch-like structures (right) with supernumerary spikelets (indicated with blue arrows) and T. sinskajae PI 418587 (a WT-spike) (left). b, Schematic representation of the WFZP gene structure and the mutation identified. The light red box indicates the AP2/ERF domain. $\mathbf{c}$, A partial $2 A^{m}$ molecular-generic map showing collocation of b $h^{m}$, wfZp-A.2 and SSR-WFZP-A (ssrCS248B13-1 from Dobrovolskaya et al. [31]) on 2A ${ }^{\mathrm{m}}$. Genetic distances are given in cM. $\mathbf{d}$-j. Scanning electron microscopy images of KT 3-24 (bh $\left.{ }^{m}\right)$ and PI 418587 (WT) inflorescences at various developmental stages. $\mathbf{d}$ and $\mathbf{e}$, Spikelet differentiation stage in the wild type (d) and $b h^{m}(\mathbf{e})$. F, Early floret differentiation stages when the spikelet meristem produces the FM in the wild type. $\mathbf{g}$ and $\mathbf{h}$, Differentiation of secondary AxMs (indicated by asterisks) in the $b h^{m}$ mutant. $\mathbf{h}$ and $\mathbf{j}$. The development of glumes $\left(\left.\mathrm{g}\right|^{*}\right)$ and $\mathrm{FM}^{*}$ s by secondary AxMs in the $b h^{m}$ mutant. i, Floret differentiation stage showing differentiated floral organs in the WT-inflorescence. $\mathbf{j}$, The development of ectopic spikelets in $b h^{m}$. Bars $=100 \mu \mathrm{m}$. $\mathbf{f}$, floret with floret organ primordia; fm, floret meristem; gl, glume; im, inflorescence meristem; l, lemma, sm, spikelet meristem; sm*, spikelet meristem of an ectopic spikelet

phenotype of line KT 3-24 resulted from the WFZP mutation. This novel allele was designated as wfzp-A.2, in accordance with $w f z p$-A.1, a frameshift mutation in bread wheat [25], and wfzp-A/TtBH-A1, a non-synonymous substitution in the AP2/ERF functional domain in T. turgidum tetraploid wheat [27].

Tetraploid T. durum wheat. R-107, a natural mutant of T. durum, is characterized by the development of a sessile SSes at spikelet nodes (under greenhouse conditions) and on extended rachilla (more frequently under field conditions). Its phenotype was similar to the horizontal spikelet phenotype of bread wheat, occurring when two or three spikelets are in a horizontal position at a spike rachis node, and to the four-rowed spike (FRS) phenotype of tetraploid T. turgidum wheat but rather different from the typical turgidum spike, which has more SSes attached to a long extended rachilla (Fig. 2a). SEM analysis of inflorescence development reveal that the first deviation from the standard scheme of development of R107 occurred at the stage when the floret meristems produced lemma primordia (Fig. 2e) in a WT spikelet, the first floral organs, in the WT-inflorescence. By contrast, in line R-107, two glume primordia become apparent (Fig. 2f), and, then secondary AxMs produced the other spikelet organs (Fig. $2 \mathrm{~h}, \mathrm{j}$ ). Therefore, ectopic spikelets developed exactly at the place of florets, as revealed in SS mutants of diploid (K-3-24) and hexaploid wheat [25]. Within a spikelet, only a basal floret(s) could be replaced by a spikelet (Fig. 2h), resulting in development of the HS supernumerary spikelet phenotype (FRS phenotype in tetraploid wheat); often, ectopic spikelets that developed distally (3rd and more distally located) did not fully develop, this arrangement also resulted in HS and FRS. We compared features of the R-107 inflorescence development with those of $T$. turgidum L. K-40750, which is characterized by the typical turgidum type of branching (Fig. 2a). In general, they were similar e.g., development of an ectopic spikelet at the location of florets at $90^{\circ}$ to WT spikelets (Fig. 2j-k) and conformed to the characteristic features of line KT-3-24 listed above (Fig. 1g, h, j).

In hexaploid, diploid (this study), and tetraploid $T$. turgidum wheats, the SS/spike branching phenotypes resulted from $w f z p$-mutations, and WFZP-A was chosen as a candidate gene for the SS phenotype of line R-107. 

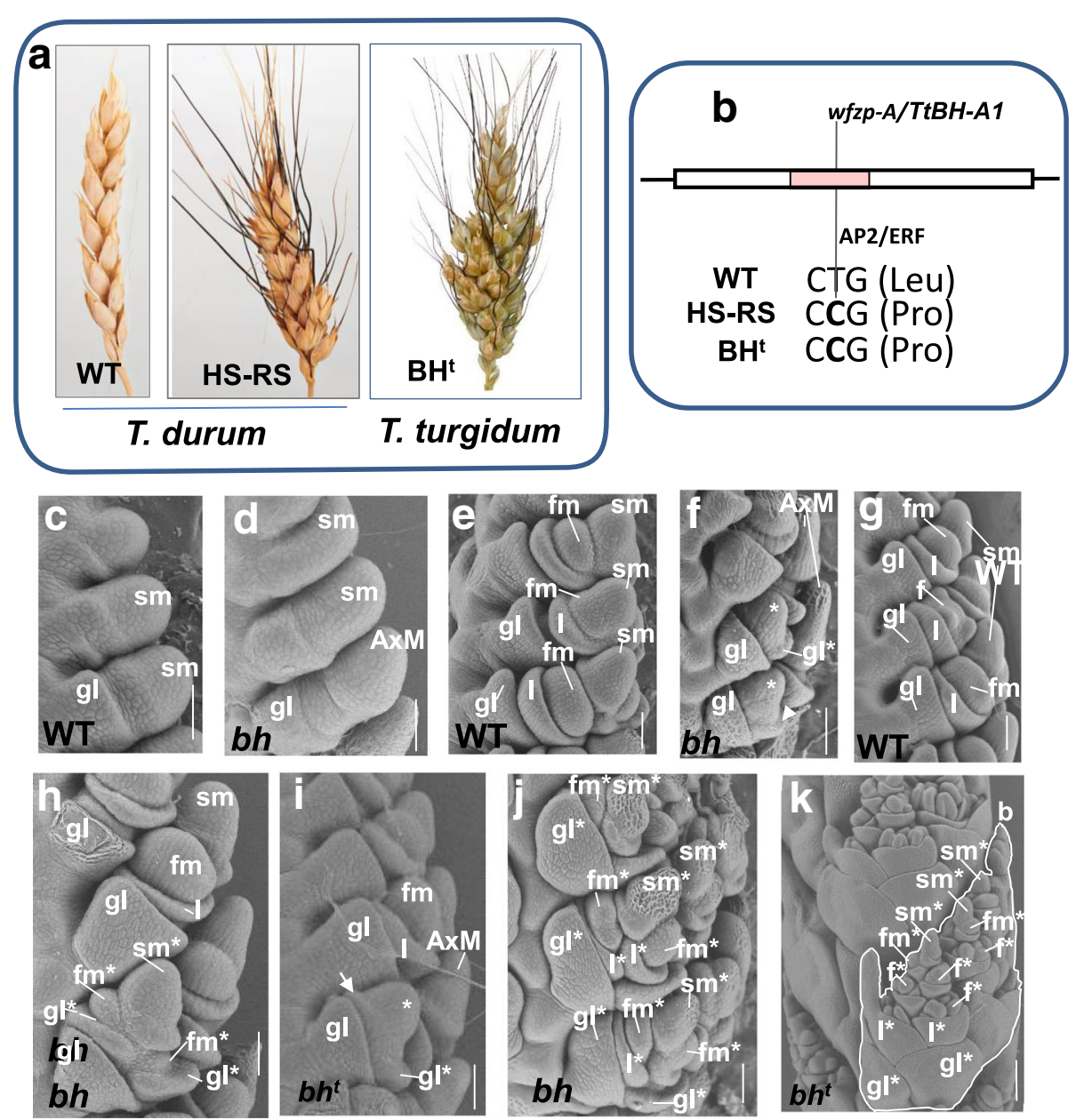

Fig. 2 Ramified spike and turgidum-type of spike branching in tetraploid wheats. a, Illustration of structure of standard spike (WT) in T. durum LD222 (left), ramified spike (indicated as gradation from horizontal spikelets, HS, to ramified spike, RS) in T. durum R-107 (middle) and the turgidum-type of spike branching (indicated as branched head, $\mathrm{BH}^{\mathrm{t}}$ ) in T. turgidum K-40750 (right). b. Schematic representation of the WFZP-A gene structures and the mutation identified. The light red box indicates the AP2/ERF domain. c-k, Scanning electron microscopy images of LD222 (WT), R-107 (bh) and K-40750 $\left(b h^{t}\right)$ inflorescences at various developmental stages. $\mathbf{c}$ and $\mathbf{d}$, Spikelet differentiation stage in the wild type (c) and bh (d). e, Early floret differentiation stages when the spikelet meristem produces FMs in WT. f, Differentiation of secondary AxMs (indicated by asterisks) in the bh mutant. $\mathbf{h}$ and $\mathbf{i}$, The development of glumes ( $\left.g\right|^{*}$, indicated by arrows) and floret meristems ( $\left.\mathrm{fm}^{*} \mathrm{~s}\right)$ by secondary AxMs in the bh and bh ${ }^{t}$ mutants. $\mathbf{g}$, Floret differentiation stage in the WT-inflorescence. $\mathbf{h}$, development of an ectopic spikelet at place of a basal floret. $\mathbf{j}$ and $\mathbf{k}$, Development of ectopic branch-like structures (b) in the $b h$ and $b h^{t}$ mutants. Bars = $100 \mu \mathrm{m}$. f, floret with floret organ primordia; fm, floret meristem; gl, glume; im, inflorescence meristem; l, lemma, sm, spikelet meristem; sm*, spikelet meristem of an ectopic spikelet

The WFZP-A gene was sequenced in line $\mathrm{R}-107$ and standard spiked LD222. We found a non-synonymous substitution, CTG (Leu) to CCG (Pro), in the AP2/ERF domain of the WFZP-A gene of line R-107. This allele is not novel and has been found in branched accessions of T. turgidum [27], near-isogenic lines that have the fourrowed spikelet FRS; and RS phenotypes of T. turgidum $[28,29]$. The WFZP-A gene was also sequenced in a branched accession T. turgidum, K-40750. As expected, $\mathrm{K}-40750$ possessed the $w f z p-A / T t B H-A 1$ mutant allele. This finding was supported by results of the complementation test: the R-107 line was crossed with K-40750, and phenotypes of the hybrids were examined. All $\mathrm{F}_{1}$ plants had the branched spike phenotype intermediate between the parental types; the $F_{2}$ plants segregated into the FRS and RS/turgidum types of branching, and both $F_{1}$ and $F_{2}$ showed the SS-phenotype.

Thus, the $w f z p$-mutations in diploid, tetraploid and hexaploid wheat species lead to similar abnormal development of the inflorescence and resulted in SS-phenotypes: MRS, HS (FRS), and RS/genuine branching/turgidum type of branching.

\section{False-true ramification of a spike}

T. turgidum PI 67339 has an SS-phenotype different from those determined by the $w f z p$ - mutations. First, the 


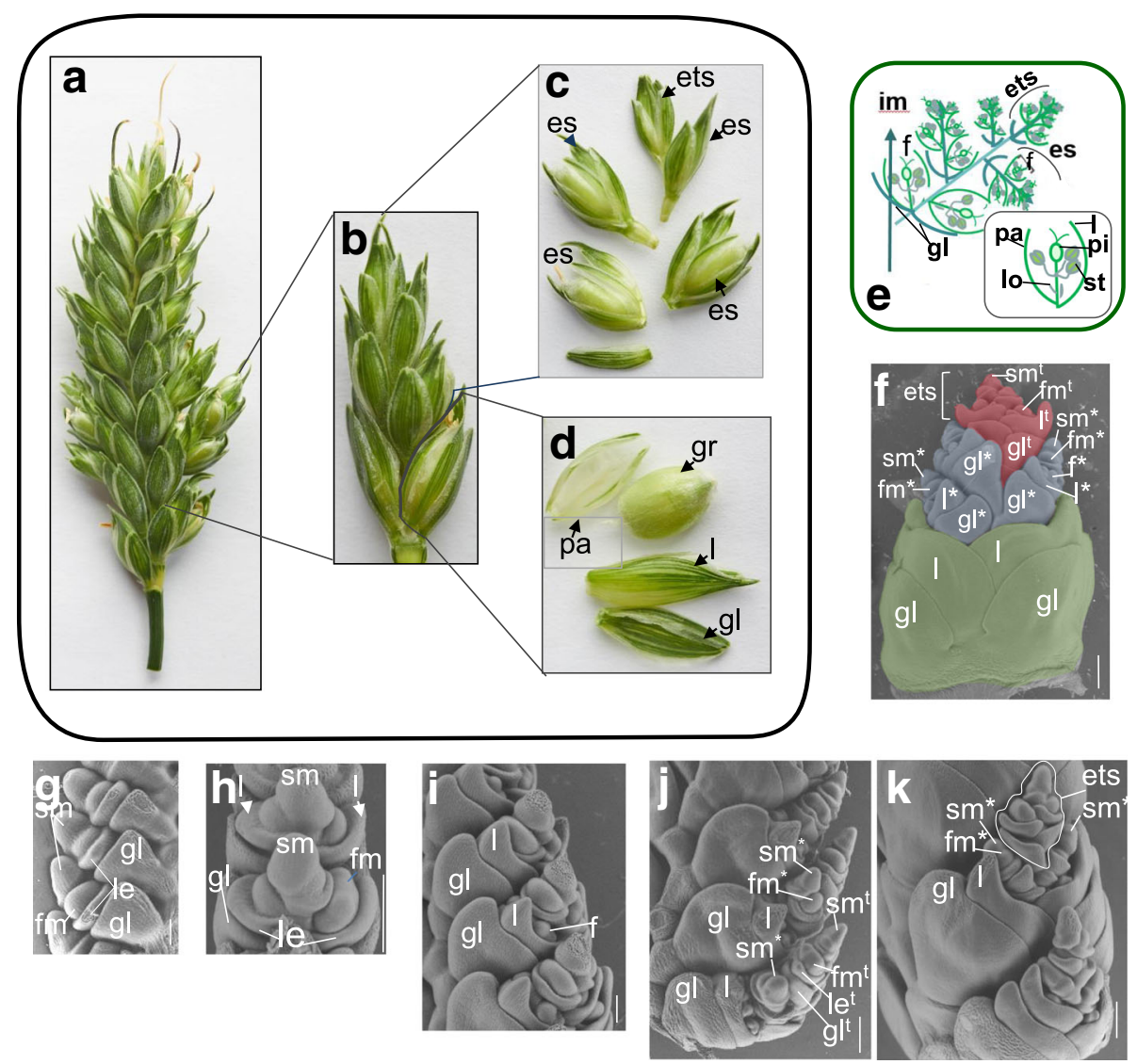

Fig. 3 False-true spike ramification in tetraploid T. turgidum wheat. a-d, Illustration of the spike structure in the T. turgidum PI 67339 (a), harboring elongated spikelets (b) with grains in the basal part (d) and ectopic spikelets in the distal part (c). e, Schematic illustration of an altered spikelet structure of the false-true spike ramification phenotype, showing locations of florets ( $f$ ), ectopic spikelets (es) and an ectopic terminal spikelet (ets), and structure of a WT-floret (right, bottom). f, Scanning electron microscopy image of an elongated spikelet dissected from the false-true ramified spike, showing the spikelet glumes ( $\mathrm{gl}$ ) and lemmas (I) of a basal pair of florets (in green), three developing ectopic spikelets at the floret differentiation stage (in gray) and an ectopic terminal spikelet (ets) at the early floret differentiation stage (in red). $\mathbf{g}$-k, SEM images of the PI 67339 inflorescence at various developmental stages. $\mathbf{g}$ and $\mathbf{h}$, Early floret differentiation stage. $\mathbf{i}$, beginning of the floret differentiation stage, when the basal floret begin to differentiate. $\mathbf{j}$ and $\mathbf{k}$, Development of ectopic spikelets in the distal part of altered spikelet. Bars $=200 \mu \mathrm{m}$. $\mathrm{f}$, floret with floret organ primordia; $\mathrm{f}^{*}, \mathrm{ft}^{\mathrm{t}}$, floret of an ectopic spikelet (es) and an ectopic terminal spikelet (ets), respectively; fm, floret meristem; fm* $\mathrm{fm}^{\mathrm{t}}$, floret meristem of es and ets, respectively; gl, glume; $\mathrm{gl}^{*}$, gl $\mathrm{l}^{\mathrm{t}}$, glume of es and ets, respectively; l, lemma; $\mathrm{l}^{*}, \mathrm{I}^{\mathrm{t}}$, lemmas of es and ets, respectively; sm, spikelet meristem; sm*, sm ${ }^{t}$, spikelet meristems of es and ets, respectively

trait was described as sham ramification (SHR), which is characterized by formation of an extended spikelet axis (rachilla) with attached florets [30]. The spike phenotype of line PI 67339 is controlled by a single recessive gene designated as shr2 (sham ramification 2) by Amagai et al. [30]. Further careful observation revealed that the SHR phenotype of PI 67339 is different from "shamramification" of T. jakubzineri, with florets at basal and apical nodes of an elongated spikelet rachilla [18]. The elongated rachilla of T. turgidum PI 67339 bears florets at basal nodes while containing spikelets at the apical nodes. Amagai et al. [18] proposed a new status of PI 67339 ramification, "false-true ramification".

Under greenhouse conditions (Novosibirsk, 2014-2017), the PI 67339 line was characterized by incomplete penetrance $(\sim 90 \%)$. In contrast, all $w f z p$-mutants of di-, tetra- and hexaploid wheat species showed $100 \%$ penetrance. We found that elongated spikelets of line PI 67339 formed at rachis nodes of the middle part of the spike (Fig. 3a). The glumes of elongated spikelets were fully developed and looked like those of WT spikelets. By contrast, glumes of branch like structures of the $w f z p$-mutants were always rudimentary ([25], results of present study). The elongated spikelet of PI 67339 was subtended by two glumes and consisted of two fertile florets that formed grains in the basal part of the spikelet, and several SSes attached to the extended spikelet rachilla (Fig. 3c-e). In these SSes, either one or two florets were fertile.

At the early stages of PI 67339 inflorescence development, the apical meristem produced primary AxMs 
(spikelet meristems) that first formed spikelet organs, glumes, and then formed secondary AxMs (Fig. 3g-h). Next, lemmas, that were first produced by secondary AxMs became visible, after which the other organs of two basal florets developed (Fig. 3i). Although the two basal florets of the elongated spikelet developed in agreement with the standard scheme of development (Fig. 2c, e, g, showing inflorescence development of line LD222: a standard spiked tetraploid wheat cultivar), further spikelet development was disrupted (altered). Secondary AxMs (correspond to the 3rd-5th floret meristems in the WT spikelet) produced glume primordia and spikelet organs at the place of florets until primary AxMs converted to the terminal spikelet meristem and formed a terminal spikelet oriented $90^{\circ}$ to the ectopic spikelets (Fig. 3f, j, k). Normally, the wheat spikelet meristem is indeterminate and continually produces floret meristems. In line PI 67339, primary axillary (spikelet) meristem first produced two florets (this situation conforms to the standard scheme of development), then produced 2-3 spikelets at the places of florets (in the way similar to that of - wfzpmutants) and finally formed a terminal spikelet at $90^{\circ}$ to previously developed ectopic spikelets.

Thus, inflorescence development of line PI 67339 has some features in common with that of $w f z p$-mutants, namely, development of ectopic spikelets at the place of the floret at $90^{\circ}$ to normal spikelets. The PI 67339 falsetrue ramification phenotype is under the control of the recessive $s h r 2$ gene, which was mapped to $2 \mathrm{AL}$ [31]. We crossed line PI 67339 (shr2) with R-107 (HS/RS phenotype under the $w f z p-A / T t B H-A 1$ control) and K-40750 (the turgidum type of spike branching under the $w f z p-A$ / $T t B H-A 1$ control) and analyzed phenotypes of $F_{1}$ and $F_{2}$ hybrids to examine a possible gene interaction.

\section{The PI $67339 \times$ R-107 cross}

$\mathrm{F}_{1}$ plants from the PI 67339/R-107 cross showed the WT spike phenotype (Fig. 4f). $F_{2}$ hybrids could be subdivided into several phenotypic classes: (\#1) the standard spike; (\#2) the HS/FRS SS-phenotype; (\#3) the true spike ramification (ramified spike, RS), when SSes developed at an extended rachilla (both \#2 and \#3 had the R-107 parental phenotype); (\#4) false-true spike ramification, (f-tR, the PI 67339 parental phenotype); (\#5) an additive phenotype, a combination of both parental phenotypes, then sessile SSes and an extended spikelet of the f-tRS type emerged at one rachis node (Fig. $4 \mathrm{~h}$; Table 2). The segregation ratio of 40 R-107 parental phenotype (BH) $F_{2}$ plants, including classes \#2, \#3, and \#5, to 103 non$\mathrm{BH} \mathrm{F}_{2}$ plants (classes \#1 and \#4) conformed to the 1:3 segregation $\left(X^{2}=0.67\right)$, which confirmed monogenic genetic inheritance of $b h$. The segregation ratio of $22 \mathrm{f}$-tR (classes \#4 and \#5) to 121 non-f-tRS deviated from 1:3 $\left(x^{2}=7\right)$. The ratio of 4 additive phenotype plants $(\# 5)$ to 139 plants of the other phenotypic classes (\# 1-4) fitted to $1: 15$ segregation $\left(x^{2}=2.7, P=0.05-0.025\right)$. These results indicated that genes $b h(w f z p-A)$ and shr2 are inherited independently and may belong to different pathways; otherwise, we would have observed dominance of one of the parental phenotypes or appearance of a new (enhanced) phenotype.

\section{The PI $67339 \times K-40750$ cross}

Although the $w f z p-A / T t B H-A 1$ mutant allele is a major genetic factor that determined the SS phenotypes of lines R-107 and K-40750, we obtained different results from the PI 67339/R-107 and PI 67339/K-40750 crosses. The $F_{1}$ hybrids from the PI $67339 /$ K-40750 cross had the PI 67339 parental spike type, f-tR, with more severe trait manifestation (Fig. 4b). $F_{2}$ hybrids segregated into several phenotypic classes (Fig. 4c-d, Table 2). In addition to the above-mentioned classes \#\#1-5, a new phenotypic class (\#6), showing spikes with extended spikelet rachilla containing numerous florets (sham ramification) was detected (Additional file 2). The segregation ratio $107 \mathrm{f}$-tR to 55 non- $\mathrm{f}$-tR deviated from 3:1 $\left(X^{2}=6.9\right)$ and indicated the dominant mode of inheritance in this cross. Segregation of $23 \mathrm{BH} \mathrm{F}_{2}$ plants (classes $\# 2$, \#3, and \#5) into 139 non- $\mathrm{BH}\left(\mathrm{X}^{2}=10.8\right)$ also deviated from 1 to 3 monogenic inheritance, and additive phenotype (Fig. 4d) was observed in 4 of $162 \mathrm{~F}_{2}$ plants, which deviated from the 1:15 segregation ratio $\left(x^{2}=3.9\right)$.

Zhang et al. [29] demonstrated that in addition to $w f z p-A / T t B H-A 1$, another genetic factor(s) is involved in the genetic control of the turgidum type of spike branching. These factors determine elongation of a spikelet rachilla and development of a RS (ramified spike). Phenotypes of lines R-107 and K-40750 differed in the length of the rachilla, K-40750 has long rachillas with numerous SSes, whereas R-107 possesses either HS/FRS or a short-rachilla (SHR) phenotype. Our results suggested that the shr2 gene may interact with the genes that participate in the development of the RS phenotype (turgidum branching), and these gene(s) may share similar functions with the shr2 gene. The presence of the additive phenotypic class ( $\mathrm{HS}$ and $\mathrm{f}$-tR) among the $\mathrm{F}_{2} \mathrm{~S}$ from the PI 67339/K40750 cross confirmed the results of the PI 67339/R-107 cross, indicating independent inheritance of shr 2 and $w f z p-A / T t B H-A 1$ and that the genes may belong to different genetic pathways.

\section{Discussion}

Wheat lines with SSes is very heterogeneous group, which comprises various non-standard morphotypes: (1) multirow spike (MRS) with numerous SSes at a rachis node; (2) horizontal spikelet (HS), including a triple 


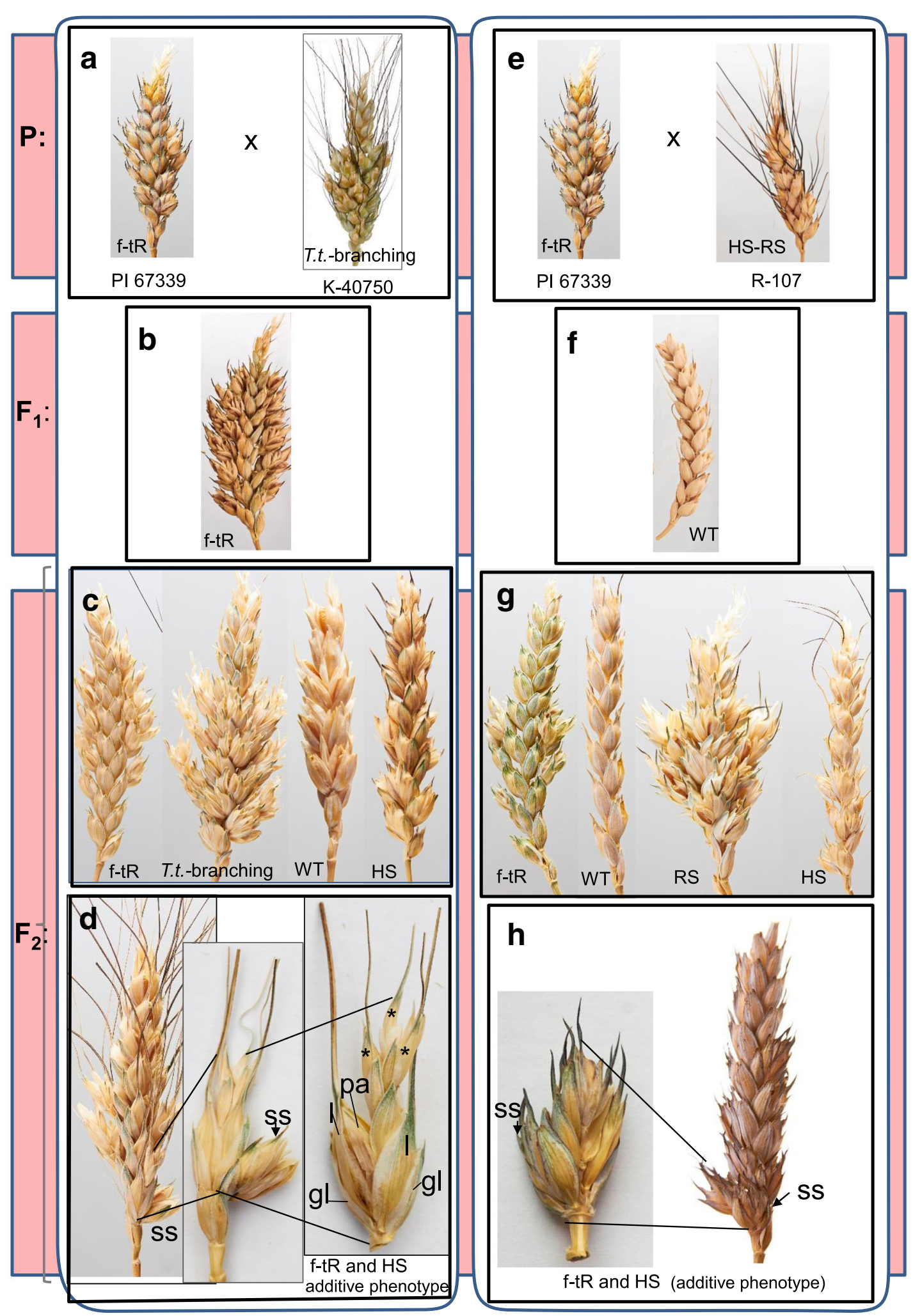

Fig. 4 (See legend on next page.) 
(See figure on previous page.)

Fig. 4 Spike phenotypes of $F_{1}$ and $F_{2}$ hybrids derived from the crosses of T. turgidum PI 67339 (shr2), T. durum R-107 (wfzp-A/ttBH-A1) and T. turgidum K-40750 (wfzp-A/TtBH-A ). a to d, spikes of the parental lines $(\mathbf{a}), F_{1}(\mathbf{b})$ and $F_{2}(\mathbf{c}$, d) hybrids derived from the PI 67339/K-40750 cross. e to $\mathbf{h}$, spikes of the parental lines $(\mathbf{e}), F_{1}(\mathbf{f})$ and $F_{2}$ hybrids $(\mathbf{g}, \mathbf{h})$ derived from the PI 67339/R-107 cross. $\mathbf{c}$ and $\mathbf{g}$, $F_{2}$-spikes, representing different phenotypic classes: false-true ramification (f-tR), turgidum type of branching (T.t.- branching), ramified spike (RS), horizontal spikelet (HS), standard or WT-spike (WT). D, $\mathrm{F}_{2}$-spike of additive phenotype (left) harboring an extra spikelet (supernumerary spikelet, SS) (middle) and an elongated spikelet of f-tR-type (right) at one rachis node. H, A spike of additive phenotype (right) with the SS and f-tR-type spikelets at one rachis node (left). gl - glumes, I - lemma, pa - palea, asterisks indicate ectopic spikelets

spikelet, and a similar phenotype in tetraploid wheat species called four rowed spikelet (FRS); (3) genuine branching (GB) or ramified spike (RS) or turgidum type of spike branching (synonyms); (4) vertical spikelets (VS) or pared spikelets, which are characterized by the formation of the second spikelet immediately adjacent to and directly below a typical single spikelet; (5) false-true spike ramification (f-t SR) with florets in the basal part and spikelets in the apical part of the elongated spikelet rachilla. Dobrovolskaya et al. [25] have demonstrated that mutations in WFZP-A, and $-D$ genes result in HS (including the triple spikelet), MRS and RS phenotypes in hexaploid wheat. Poursarebani et al. [27] have shown that the TtBH-A1 mutation (a mutation at the WFZP-A gene locus) is responsible for development of the turgidum-type of spike branching in tetraploid T. turgidum wheat. Zhang et al. $[28,29]$ have developed near isogenic lines with FRS and long ramified spike phenotypes based on a cross of branched T. turgidum and standard spiked lines, and demonstrated that, in addition to TtBH-A1, other genetic factors are involved in the genetic control of the SS phenotype with the extended rachilla, including the RSphenotype. Among these factors, Zhang highlighted a dominant gene that is also localized to chromosome $2 \mathrm{~A}$ $[28,29]$. Although $w f z p$-mutations are responsible for MRS, HS, triple-spikelet, and RS (GB) SS phenotypes, the VS or pared-spikelet phenotype is under the control of another gene. Boden et al. [34] have revealed that Photoperiod-1 (Ppd-1), a pseudo-response regulator gene that controls photoperiod-dependent floral induction, has a major inhibitory effect on paired spikelet formation by regulating the expression of FLOWERING LOCUS T (FT).

In the present study, we examined an induced SSmutant with the branched spike (development of SSes on short branches) in diploid wheat T. monococcum and

Table 2 Segregation data in $F_{2}$ populations

\begin{tabular}{llllllll}
\hline Cross & $\mathrm{F}_{2}$ & & & & & & \\
\cline { 2 - 8 } & NS & HS (FRS) & RS & f-tR & HS and f-tR & SHR & Total \\
& $(\# 1)$ & $(\# 2)$ & $(\# 3)$ & $(\# 4)$ & $(\# 5)$ & $(\# 6)$ & \\
\hline Pl67339/K40750 & 26 & 7 & 12 & 103 & 4 & 10 & 162 \\
Pl67339/R-107 & 85 & 32 & 4 & 18 & 4 & - & 143 \\
\hline
\end{tabular}

HS horizontal spikelet, $F R S$ four-rowed spike, $R S$ ramified spike, $f$-tR false-true ramification, NS normal spike, $\mathrm{HS}$ and f-tR additive phenotype, $S H R$ sham ramification (plants showing extended spikelet rachilla harboring florets) found that the mutant phenotype resulted from a missense mutation in the AP2/ERF functional domain of WFZP-A. This novel allele was designated as $w f z p-A .2$. Spontaneous mutants with spike branching have not been found in diploid wheat species [9].

At the tetraploid level, T. turgidum forms with spikebranching have been well known for a long time [4]. Poursarebani et al. [28] demonstrated that the phenotype of 30 branched $T$. turgidum accessions is associated with the same mutant allele TtBH-A1 at the WFZP-A gene locus; this finding is suggestive of this allele's monophyletic origin. Distribution areas and ecological characteristics of unbranched T. turgidum convar. turgidum and branched $T$. turgidum convar. compositum (L.f.) Filat. accessions in general coincide; with an exception of the Tibetan ecotype, all accessions of this ecotype are unbranched [4]. Consequently, the TtBH-A1 mutation may have arisen early in the T. turgidum evolutionary history and then has been spread across modern habitats of this species.

On the other hand, in $T$. durum tetraploid wheat, branched spiked forms are rare [4]. In 1952, M.M. Jakubziner found branched forms in two cultivars of T. durum: Akmolinka 5 and Hordeiforme; these forms were designated as ramosohordeforme and ramosoapulicum [8]. Two original forms of durum wheat with a branched spike, var. ramosohordeforme and var. ramosoapulicum, were found by V.F. Dorofeev during an expedition to Transcaucasia (1961-1964) under the natural conditions of wheat growing in local mixed populations, where bread wheat, $T$. durum and T. turgidum were grown side by side [8]. Dorofeev suggested that the branched forms of T. durum might have originated from spontaneous hybridization between branched T. turgidum accessions and standard spiked T. durum, which were grown side by side in Transcaucasia. We characterized the spontaneous SS mutant of T. durum, R-107, which was found in Dagestan, Russia, and demonstrated that its mutant phenotype is determined by the $w f z p-A / T t B H-A 1$ mutant allele, which is responsible for the branching spike phenotype of $T$. turgidum convar. compositum (L.f.) Filat. This finding confirmed Dorofeev's assumption that the branched phenotype of $T$. durum grown in Transcaucasia - or in any regions where $T$. turgidum and other wheat species crossable with $T$. turgidum grow side by side may have originated from $T$. turgidum via spontaneous hybridization. 
It should be noted that the R-107 line showed a phenotype that is more similar to FRS than to the typical turgidum spike branching type. This finding means that the genetic background is important for expression of the turgidum type of spike branching.

Now that the wfzp mutants were characterized in detail in di-, tetra-, and hexaploid wheats, we can make some conclusions regarding common characteristics of their phenotypes and developmental features that can help to predict whether any new uncharacterized SSmutant phenotype may be a result of a $w f z p$-mutation. Even though wfzp mutations caused several SS morphotypes mentioned above, all these morphotypes have common characteristics: location of ectopic spikelets at the place of florets at $90^{\circ}$ to a WT-spikelet or an ectopic spikelet of the previous order as well as formation of rudimentary glumes that are visible only at the early stages of development. Under the influence of weak $w f z p$ mutations, only one SS develops at a rachis node of the lower part of a spike [25, 35], and these spikelets are always positioned at an angle to a WT-spikelet. It is also noteworthy that both weak and severe $w f z p$ mutations cause replacement of basal florets by ectopic spikelets, whereas more distally located florets within the altered spikelet may develop normally in weak $w f z p$ mutants $[25,36]$. Independently of the ploidy level, all the characterized $w f z p$-mutants of wheat were fertile.

The phenotype of $w f z p$ mutants is similar to phenotypes of barley com2 [27], Brachypodium moc1 [36], rice fzp [37] and maize $b d 1$ [38] mutants. The expression pattern and function predicted by means of $f z p$-mutant phenotypes were similar. Bai et al. [39] demonstrated that in rice $F Z P$ plays an important role in the regulation of $A B C D E$ floral organ identity genes. FZP controls panicle branching and spikelet formation by regulating RFL/ABERRANT PANICLE ORGANIZATION 2 (APO2). Overexpression of $F Z P$ severely represses AxM formation and outgrowth of secondary branches in the panicle, and it positively regulates the expression of a subset of the class $B$ and $E$ genes, suggesting that FZP may specify floral organ identity by regulating the related OsMADS-box genes [39].

At present, $w f z p$-mutants and VS lines are the most studied among SS-wheats [25, 27, 34], whereas sham ramification and false-true spike ramification traits are not well studied so far. False-true ramification line PI 67339 was examined here by SEM analysis and a classical genetic approach. We found that inflorescence development of the PI 67339 line and that of SS lines, determined by wfzp mutations, have some common features, namely, development of ectopic spikelets (at the place of florets), which are oriented at $90^{\circ}$ to WT-spikelets. Nonetheless, the earliest events during spikelet development of these two morphotypes were different: in PI 67339, development of the $1^{\text {st }}$ and $2^{\text {nd }}$ florets conforms the standard scheme of wheat inflorescence development, but in the $w f z p$-mutants, abnormal development was observed earlier, and ectopic spikelets developed always at the place of floret(s) in the basal part of a spikelet. The FZP gene and its orthologs in cereals participate in the establishment of floral meristem identity, and $f z p$ mutations affect early events during spikelet development [25, 27, 36-38]. The shr2 mutant phenotype suggests that $S H R 2$ is required for establishing floret meristems at a later stage of spikelet development, during the development of the $3^{\text {rd }}$ to $5^{\text {th }}$ florets. Results of tests for gene interaction, namely, the presence of an additive phenotype in $\mathrm{F}_{2}$ hybrid populations produced by crosses of R-107 (wfzp-A/bh1), K-40750 (wfzp-A/bh1), and PI 67339 (shr2), suggest that the WFZP and SHR2 genes function independently in different processes during spikelet development.

Wheat has indeterminate spikelet meristems, SMs, and the wheat spikelet carries multiple florets. The number of fertile florets per spikelet was determined by the number of floret primordia and of florets with hypoplasia. Indeterminacy of the SM is not affected by polyploidization and is a characteristic feature of the spikelet of di-, tetra- and hexaploid wheats [40]. In florets of the distal portion of the spikelet, two types of hypoplasia have been observed: (1) floret organs are differentiated, but they are sterile and aborted (e.g., tetra- and hexaploid wheats), or (2) floret meristem initiates but no floral organs develop (in diploid wheat) [40]. In $w f z p$-mutants, the primary AxM (corresponds to SM in a WT spikelet) is indeterminate and produces numerous secondary AxMs (correspond to FMs in a WT-spikelet) that either form ectopic spikelets or, due to hypoplasia in the distal part of a branch-like structure, remain underdeveloped. Indeterminacy is a characteristic feature of branch-like structures of $f z p$ mutants in wheat $(w f z p)$, barley $(\operatorname{com} 2)$, rice $(f z p)$, and maize $(b d 1)$. Nonetheless, in the false-true spike ramification line PI 67339, the altered extended spikelet is determinate and possesses an ectopic terminal spikelet that developed at $90^{\circ}$ to the other ectopic spikelets; this arrangement suggests that mutations in the SHR2 gene may alter the SM fate. Although the shr2 gene has not been cloned yet, we can suggest its function(s) on the basis of its mutant phenotype and the features of inflorescence development. The gene may be involved in the maintenance of floral meristem identity during spikelet development, when the $3^{\text {nd }}-4^{\text {th }}\left(5^{\text {th }}\right)$ florets differentiate; moreover, shr 2 may determine the spikelet meristem fate. Our results suggest that WFZP and SHR2 are independently inherited and probably "sit" on different regulatory pathways, whereas still unidentified gene(s) that are involved in the control of the turgidum spike branching phenotype in addition to the major wfzp$A / T t B H-A 1$ gene may interact with shr2 and share some functions in the inflorescence development. 


\section{Conclusions}

Accordingly, SS mutants represent an important genetic tool for research on the development of the wheat spikelet and for identification of genes that control meristem activities. Further studies on different non-standard SS morphotypes and wheat lines with altered spike morphology will allow researchers to identify new genes that control meristem identity and determinacy, to elucidate the interaction between the genes and to understand how these genes, acting in concert, regulate the development of the wheat spike. SS wheats lines have been attracting attention of breeders for many years; but their potential has not been realized yet.

\section{Additional files}

Additional file 1: Sequences of WFZP-A of diploid and tetraploid wheat species. (PDF $43 \mathrm{~kb}$ )

Additional file 2: Spike with extended spikelet rachilla containing numerous florets. (PDF $170 \mathrm{~kb}$ )

\section{Acknowledgments}

We thank Dr. Mitrofanova (N.I. Vavilov All-Russian Institute of Plant Genetic Resources (St. Petersburg, Russia) for providing T. turgidum accessions, including K-40750, Ms.Oxana A. Roschina (Institute of Cytology and Genetics SB RAS, Novosibirsk, Russia) for technical assistance.

\section{Funding}

This work was supported by grants of Russian Foundation for Basic Research (Projects 15-04-05371 and 16-54-53064) and the IC\&G Budgetary Project no. 0324-2016-0001. Re-sequencing of the WFZP-A gene was supported by RSF (N 16-16-10021). P. M. thanks for projects Nos. QJ1510206 and R01115 of the Ministry of Agriculture of the Czech Republic. Publication costs were funded by Russian Foundation for Basic Research (Project 15-04-05371).

\section{Availability of data and materials}

All data generated or analyzed during this study are included in this published article [and its supplementary information files].

\section{About this supplement}

This article has been published as part of BMC Plant Biology Volume 17 Supplement 2, 2017: Selected articles from Belyaev Conference 2017: plant biology. The full contents of the supplement are available online at https:// bmcplantbiol.biomedcentral.com/articles/supplements/volume-17-supplement-2.

\section{Authors' contributions \\ OBD designed and managed the project. OBD and NW designed and performed genetic experiments. YA participated to the genotyping. AAK, AED and OBD contributed to the SEM analysis. YA and CIP carried out re- sequencing. PM contributed to the writing of the manuscript. OBD wrote the manuscript with input from all other coauthors. All authors read and approved the final manuscript.}

\section{Ethics approval and consent to participate}

Not applicable.

\section{Consent for publication}

Not applicable.

\section{Competing interests}

The authors declare that the research was conducted in the absence of any commercial or financial relationships that could be construed as a potential conflict of interests.

\section{Publisher's Note}

Springer Nature remains neutral with regard to jurisdictional claims in published maps and institutional affiliations.

\section{Author details}

${ }^{1}$ Institute of Cytology and Genetics, SB RAS, Lavrenvieva ave. 10, Novosibirsk 630090, Russia. ${ }^{2}$ Novosibirsk State University, Pirogova, 2, Novosibirsk 630090, Russia. ${ }^{3}$ College of Agriculture, Ibaraki University, Ibaraki, Japan. ${ }^{4}$ Agrotest Fyto, Ltd., Kroměříž, Czech Republic. ${ }^{5}$ Central Siberian Botanical Garden SB RAS, Novosibirsk, Russia.

Published: 28 December 2017

\section{References}

1. Malcomber ST, Preston JC, Reinheimer R, Kossuth J, Kellogg EA Developmental gene evolution and the origin of grass inflorescence diversity. In: Soltis DE, Soltis PS, Leebens-Mack J, editors. Developmental genetics of the flower, advances in botanical research. Elsevier Itd; 2006. p. 423-79.

2. Muramatsu MA. Presumed genetic system determining the number of spikelets per rachis node in the tribe Triticeae. Breed Sci. 2009;59:617-20.

3. Sakuma S, Salomon B, Komatsuda T. The domestication syndrome genes responsible for the major changes in plant form in the triticeae crops. Plant Cell Physiol. 2011;52:738-49.

4. Dorofeev VF, Korovina ON. Cultivated Flora of the USSR.V.1. Wheat. Leningrad: Kolos Publ; 1979. (in Russian)

5. Dahlgren BE. Wheat. Chicago: field museum of. Nat Hist. 1922.

6. Percival J. The wheat plant. London: Duckworth and Co; 1921.

7. Coffman FA. Supernumerary spikelets in Mindum wheat. J Hered. 1924:5:187-92.

8. Dorofeev VF. Branched forms of durum wheat. Selekzia i semenovodstvo. 1969:2:78-9. (in Russian)

9. Goncharov NP. Comparative genetics of wheats and their related species. In: Editor: V. K. Shumny. Edition: 2nd, Publisher: Academic Publ. House "GEO", Novosibirsk. 2012. (in Rus).

10. Amagai $Y$, Martinek $P$, Watanabe N, Kuboyama T. Microsatellite mapping of genes for branched spike and soft glumes in Triticum monococcum L. Genet Resour Crop Evol. 2014;61:465-71.

11. Sharman BL. Branched head in wheat and wheat hybrids. Nature. 1944;153:497-8.

12. Swaminathan MS, Chopra VL, Sastry GRK. Expression and stability of induced mutation for branching in bread wheat. Curr Sci. 1966;35:91-2.

13. Melnik VM. Genetic analysis of sphaerococcoid mutant of common wheat Triticum aestivum L. In: Selektsiya Selskhozyaistvennykh Kultur v Altayskom Kraye. SO VASKHNIL, Novosibirsk: Altai Agricultural \& Breeding Research Institute; 1988. p. 59-70. (in Russian).

14. Li J, Wang Q, Wei H, Hu X. Yang W. SSR mapping for locus conferring on the triple spikelet trait of the Tibetan triple-spikelet wheat (Triticum aestivum L. concv. Tripletum). Triticeae Genomics Genet. 2011;2:1-6.

15. Martinek P. Gene resources with non-standard spike morphology in wheat. Proceedings of the 9th International Wheat Genetics Symposium. 1998; 2: 286-288

16. Martinek P, Bednar J. Changes of spike morphology (multirow spikeMRS, long glumes-LG) in wheat (Triticum aestivum L.) and their importance for breeding. In: The proceedings of international conference "genetic collections, isogenic and alloplasmic lines", Novosibirsk, Russia, 2001 pp 192-194.

17. Klindworth DL, Williams ND, Joppa LR. Inheritance of supernumerary spikelets in a tetraploid wheat cross. Genome. 1990;33:509-14.

18. Amagai Y, Gowayed S, Martinek P, Watanabe N. The third glume phenotype is associated with rachilla branching in the spikes of tetraploid wheat (Triticum L.). Genet Resour Crop Evol. 2017. doi:10.1007/s10722-017-0503-7.

19. Pennell AL, Halloran GM. Inheritance of supernumerary spikelets in wheat. Euphytica. 1983:32:767-76.

20. Dobrovolskaya O, Martinek P, Voylokov AV, Korzun V, Röder MS, Börner A. Microsatellite mapping of genes that determine supernumerary spikelets in wheat (T. aestivum) and rye (S. cereale). Theor Appl Genet. 2009;119:867-74.

21. Haque MA, Martinek P, Kobayashi S, Kita I, Ohwaku K, Watanabe N, Kuboyama T. Microsatellite mapping of genes for semi-dwarfism and branched spike in Triticum durum Desf. Var. ramosoobscurum Jakubz. "Vetvistokoloskaya". Genet Resour Crop Evol. 2012;59:831-7. 
22. Klindworth DL, Williams ND, Joppa LR. Chromosomal location of genes for supernumerary spikelets in tetraploid wheat. Genome. 1990;33:515-20.

23. Klindworth DL, Klindworth MM, Williams ND. Telosomic mapping of four genetic markers in durum wheat. J Hered. 1997;88:229-32.

24. Yang $W-Y$, Lu B-R, Hu X-R, Yu Y, Zhang Y. Inheritance of the triple-spikelet character in a Tibetan landrace of common wheat. Genet Resour Crop Ev. 2005;52:847-51.

25. Dobrovolskaya O, Pont C, Sibout R, Martinek P, Badaeva E, et al. FRIZZY PANICLE drives supernumerary spikelets in bread wheat. Plant Physiol. 2015;167:189-99.

26. Echeverry-Solarte M, Kumar A, Kianian S, Mantovani EE, Simsek S. et al, Genome-wide genetic dissection of supernumerary spikelet and related traits in common Wheat. Plant Gen. 2014;7.

27. Poursarebani N, Seidensticker T, Koppolu R, Trautewig C, Gawronski P, et al. The genetic basis of composite spike form in barley and 'miracle-wheat'. Genetics. 2015;201:155-65.

28. Zhang W, Li A, Tian J, Zhao L. Development of near-isogenic lines of wheat carrying different spike branching genes and their agronomic and spike characters. J Agric Sci. 2012;4:215-21.

29. Zhang RQ, Hou F, Chen J, Chen SL, Xing LP, Feng YG, Cao AZ. Agronomic characterization and genetic analysis of the supernumerary spikelet in tetraploid wheat (Triticum turgidum L.). J Integrative Agr. 2017;16:1304-11.

30. Amagai Y, Aliyeva AJ, Aminov NK, Martinek P, Watanabe N, Kuboyama T. Microsatellite mapping of the genes for sham ramification and extra glume in spikelets of tetraploid wheat. Genet Resour Crop Evol. 2014;61:491-8.

31. Dobrovolskaya OB, Pont C, Orlov YL, Salse J. Development of new SSR markers for homoeologous WFZP gene loci based on the study of the structure and location of microsatellites in gene-rich regions of chromosomes 2AS, 2BS, and 2DS in bread wheat. Russian J Genet: Applied Res. 2016;6:330-7.

32. Bonnett OT. The development of the wheat spike. J Agr Res. 1936;53:445-51.

33. Murai K, Takumi S, Koga H, Ogihara Y. Pistillody, homeotic transformation of stamens into pistil-like structures, caused by nuclear-cytoplasm interaction in wheat. Plant J. 2002;29:169-81.

34. Boden SA, Cavanagh C, Cullis BR, Ramm K, Greenwood J, et al. Ppd-1 is a key regulator ofinflorescence architecture and paired spikelet development in wheat. Nature Plants. 2015:14016.

35. Dobrovol'skaia OB, Badaeva ED, Adonina IG, Popova OM, Krasnikov AA, et al. Investigation of morphogenesis of inflorescence and determination of the nature of inheritance of "supernumerary spikelets" trait of bread wheat (Triticum aestivum L.) mutant line. Russ J Dev Biol. 2014;45:361-6.

36. Derbyshire P, Byrne ME. MORE SPIKELETS 1 is required for spikelet fate in the inflorescence of Brachypodium. Plant Physiol. 2013;161:1291-302.

37. Komatsu M, Chujo A, Nagato Y, Shimamoto K, Kyozuka J. FRIZZY PANICLE is required to prevent the formation of axillary meristems and to establish floral meristem identity in rice spikelets. Development. 2003;130:3841-50.

38. Chuck G, Muszynski M, Kellogg E, Hake S, Schmidt RJ. The control of spikelet meristem identity by the branched silkless 1 gene in maize. Science. 2002; 298:1238-41.

39. Bai $X$, Huang $Y$, Mao $D$, Wen $M$, Zhang $L$, Xing $Y$. Regulatory role of FZP in the determination of panicle branching and spikelet formation in rice. Scientific reports. 2016;6:19022 | doi: 10.1038/srep190221.

40. Shitsukawa N, Kinjo H, Takumi S. Murai K. Heterochronic development of the floret meristem determines grain number per spikelet in diploid, tetraploid and hexaploid wheats. Ann Bot. 2009;104:243-51.

\section{Submit your next manuscript to BioMed Central and we will help you at every step:}

- We accept pre-submission inquiries

- Our selector tool helps you to find the most relevant journal

- We provide round the clock customer support

- Convenient online submission

- Thorough peer review

- Inclusion in PubMed and all major indexing services

- Maximum visibility for your research

Submit your manuscript at www.biomedcentral.com/submit
Biomed Central 